\title{
Patulous Eustachian Tube Obliteration Using Endovascular Coils: A Novel Technique
}

\section{Introduction:}

- Patulous Eustachian tube (PET) is a condition in which the eustachian tube (ET) remains patent leading to symptoms of autophony and aural fullness.

- There is a female predominance, with an incidence of $0.3-6.6 \%$, and a mainly idiopathic aetiology.

- Other causes include rapid weight loss in bariatric surgery or pregnant patients, muscular atrophy related to neurological pathology and medications for example. diuretics..$^{1,2}$

- Medical therapy includes conservative management and nasal preparations, with those resistant to medical therapy undergoing surgical intervention.

- Various surgical modalities are described within literature, ranging from grommets to autologous fat injections into the ET cushions.

- We describe a novel surgical technique and provide a review of current alternative surgical management.

\section{Review of Current Management:}

- Method 1: A prospective trial of 11 patients analysing submucosal graft implantation was shown to provide lasting relief of symptoms (15.8 months follow up) and a $7 \%$ failure rate. ${ }^{2}$

- Method 2: A new approach using laser assisted curvature inversion technique in 11 patients provided a success rate of $81.8 \%$ with a 24 month mean follow up. ${ }^{3}$

- Method 3: A retrospective review of 52 PET patients whom underwent nasal instillation of saline showed a $63.5 \%$ success rate, more in males than females. ${ }^{4}$

- Method 4: Botulinium toxin A injected into the paratubal muscles, in a professional musician whom was symptomatic for 20 years, provided symptomatic relief for 9 months. ${ }^{5}$

- Method 5: A further series reports ligation of the pharyngeal orifice of the ET in 15 patients. Over half of the patients reported a good to excellent outcome. ${ }^{6}$

- Method 6: Occlusion of the ET by insertion of a silicone plug through a transtympanic incision to the tympanic end of the ET to obstruct the isthmus provided a $71.4 \%$ success rate in 42 ears (mean follow up 38.9 months). ${ }^{7}$

- Method 7: Another report involved 2 cases of autologous fat grafting after cauterisation of the $E T$ at the nasopharyngeal opening with myringotomy and placement of grommet (1 year follow up) - aiming for permanent stenosis of the $\mathrm{ET}^{8}{ }^{8}$

- Method 8: An update on the condition in 2005 showed that an endoscopic approach to seal tubal lumen was effective but further research was needed. ${ }^{9}$

\section{Surgical Technique:}

- Equipment required:

a) Standard Functional Endoscopic Sinus Surgery set.

b) 70 degree Relieva sinus guide catheter from a balloon sinuplasty set.

c) 0 degree rigid endoscope

d) $3 \times 3.3 \mathrm{~mm}$ VortXTM Diamond coil.

- The ET patency is confirmed with a light pipe from sinuplasty set and an assessment of the tympanic membrane is made using an operating microscope.

- The $3 \times 3.3 \mathrm{~mm}$ VortX'M Diamond (fibered platinum coil) coil is deployed $1.5 \mathrm{~cm}$ from the ET orifice in Ipost nasal space (under guidance by a consultant neuro-radiologist.)

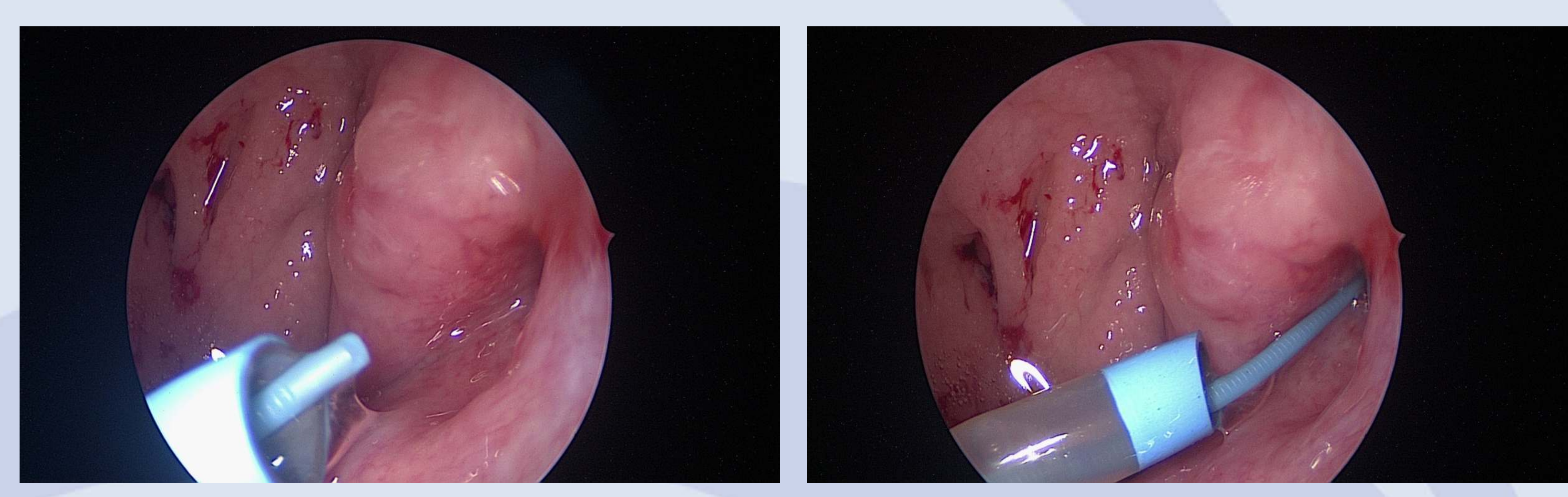

Figures 1 and 2: Endoscopic guided insertion of guide wire into the left ET

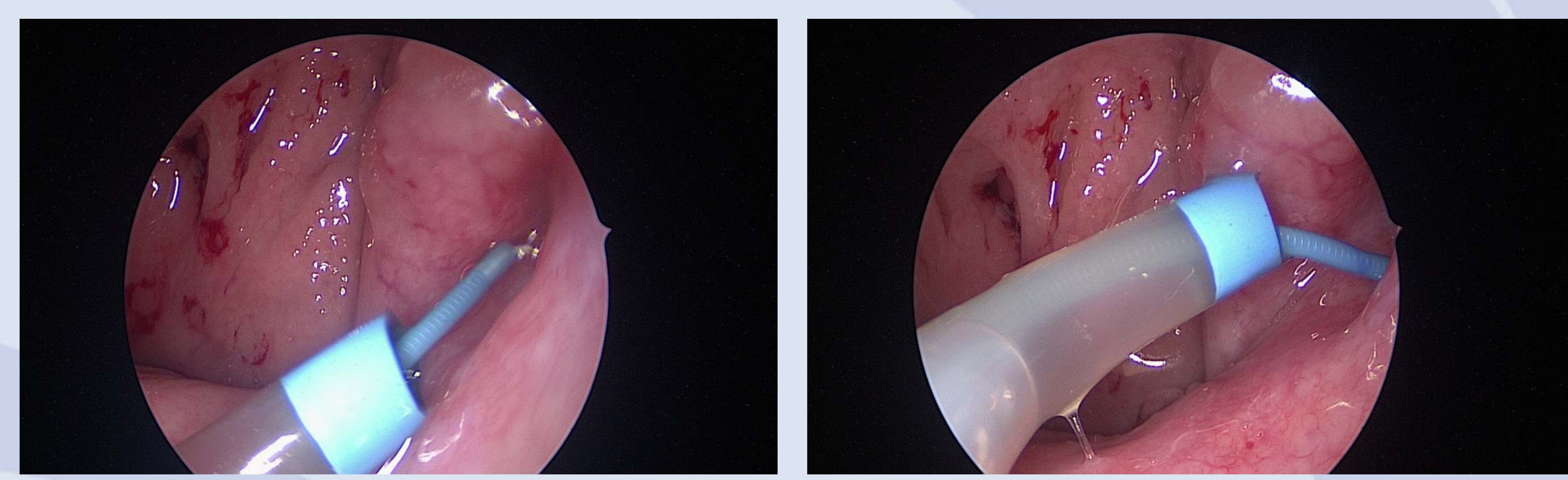

Figures 3 and 4: Marker at $1.5 \mathrm{~cm}$ and VortX ${ }^{T M}$ Diamond coil being deployed

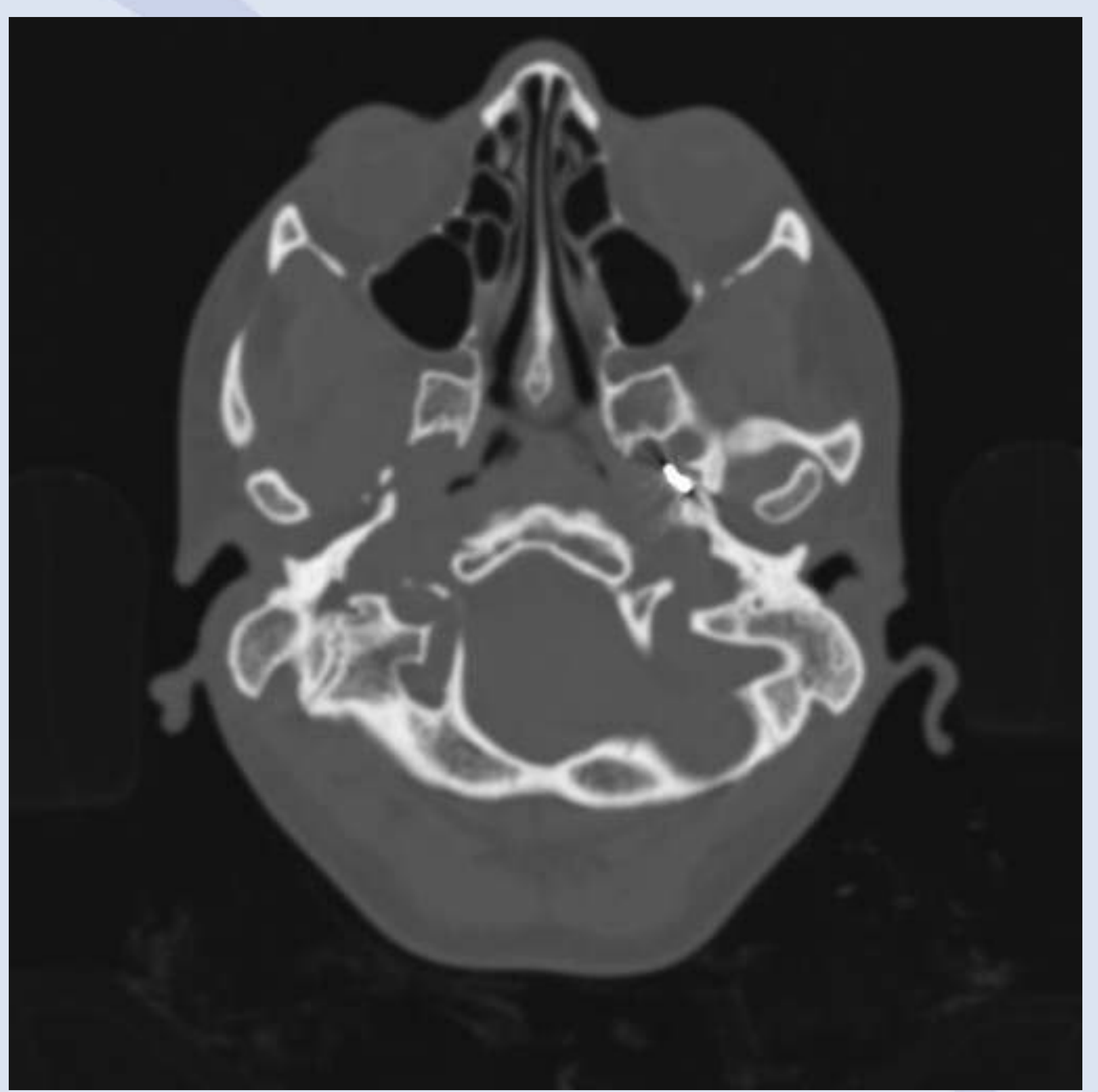

Figure 5: Immediate post-operative CT performed to confirm pla

\section{Future Aims:}

- No Cochrane review or guidelines are available and many of the case series have small numbers.

- Although our limited series have demonstrated positive outcomes to date, a larger case series is required to permit comparison of long-term outcomes with current methods described within literature.

- Further extensive research and trials are required to demonstrate significant benefit, although early follow-ups have been favourable.

\section{References:}

1. www.emedicine.medscape.com

2. Diagnosis and management of the patulous eustachian tube. Poe DS. Otology \& Neurotology. 28(5):668-77, 2007 Aug.

3. Curvature inversion technique: a novel tuboplastic technique for patulous Eustachian tube--a preliminary report. Yanez C. Pirron JA. Mora N. Otolaryngology - Head \& Neck Surgery. 145(3):446-51, 2011 Sep.

4. Nasal instillation of physiological saline for patulous eustachian tube. Oshima T. Kikuchi T. Kawase T. Kobayashi T. Acta Oto-Laryngologica. 130(5):550-3, 2010 May.

5. Successful treatment of autophonia with botulinum toxin: case report. Olthoff A. Laskawi R. Kruse E. Annals of Otology, Rhinology \& Laryngology. 116(8):594-8, 2007 Aug.

6. Ligation of eustachian tube for intractable patulous eustachian tube: a preliminary report. Takano A. Takahashi H. Hatachi K. Yoshida H. Kaieda S. Adachi T. Takasaki K. Kumagami H. Tsukasaki N. European Archives of Oto-Rhino-Laryngology. 264(4):353-7, 2007 Apr.

7. Trans-tympanic silicone plug insertion for chronic patulous Eustachian tube. Sato T. Kawase T. Yano H. Suetake M. Kobayashi T. Acta Oto-Laryngologica. 125(11):1158-63, 2005 Nov.

8. Autologous fat grafting for the refractory patulous eustachian tube. Doherty JK. Slattery WH 3rd. Otolaryngology - Head \& Neck Surgery. 128(1):88-91, 2003 Jan.

9. Update on eustachian tube dysfunction and the patulous eustachian tube. Grimmer JF. Poe DS. Current Opinion in Otolaryngology \& Head \& Neck Surgery. 13(5):277-82, 2005 Oct. 